
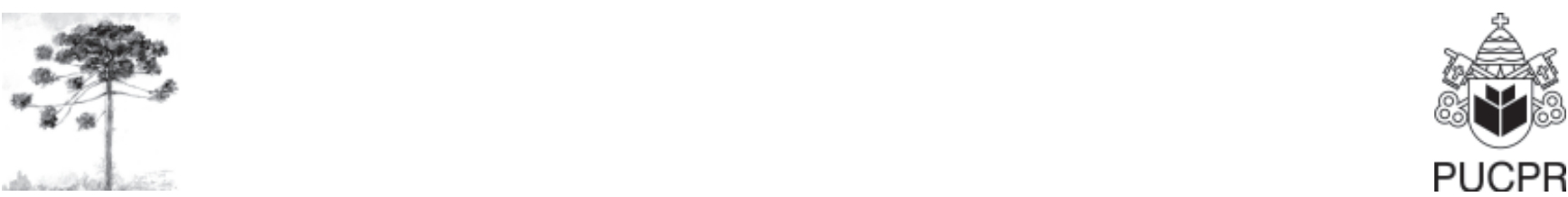

\title{
OCORRÊNCIA DE METACERCÁRIAS DE Ithyoclinostomum dimorphum EM TRAÍRAS NO RIO GRANDE DO SUL, BRASIL: relato de caso
}

\author{
Occurrence of Metacercaries of Ithyoclinostomum dimorphum in Trairas \\ at the Rio Grande do Sul State, Brazil: case report
}

\author{
Miguel Gallio ${ }^{[1]}$, Aleksandro Schafer da Silva ${ }^{[1]}$, João Fabio Soares ${ }^{[1]}$, \\ Marcos Kipper da Silva ${ }^{[1]}$, Edeson Luis Salomão ${ }^{[2]}$, Silvia Gonzalez Monteiro ${ }^{[3]}$ \\ ${ }^{[1]}$ Graduado em Medicina Veterinária pela Universidade Federal de Santa Maria (UFSM), Santa Maria, RS - Brasil. \\ ${ }^{[2]}$ Médico Veterinário Autônomo. \\ ${ }^{[3]}$ Professor adjunto do Departamento de Microbiologia e Parasitologia, Centro de Ciências da Saúde da Universidade Federal \\ de Santa Maria (UFSM), Santa Maria, RS - Brasil, e-mail: sgmonteiro@uol.com.br
}

\section{Resumo}

Ithyoclinostomum dimorphum é um trematoda, digenético, da família Clinostomidae que parasita aves aquáticas e peixes. O objetivo deste trabalho foi relatar o parasitismo por I. dimorphum em traíras da espécie Hoplias malabaricus na região central do Rio Grande do Sul, Brasil. Foram necropsiados dois exemplares oriundos de um lago no município de Cachoeira do Sul (Zoológico), nos quais foram encontrados no interior da musculatura cistos pretos. No interior de cada cisto, havia uma metacercária, que foi coletada, fixada em AFA e corada, para posterior montagem em lâminas permanentes com bálsamo do Canadá. As metacercárias foram identificadas como pertencentes à espécie $I$. dimorphum. O parasito adulto é encontrado em aves em região Sul e Centro-Oeste do Brasil. Provavelmente esses peixes foram infectados pela presença de aves migratórias no lago do zoológico em alguns períodos do ano. Pelas circunstâncias, conclui-se que o I. dimorphum é um parasita de traíras.

Palavras-chave: Hoplias malabaricus; Ithyoclinostomum dimorphum; Parasitismo. 


\section{Abstract}

Ithyoclinostomum dimorphum is a trematode, digenetic, of the Clinostomidae family that parasitize aquatic birds and fishes. The objective of this paper was to relate the parasitism for I. dimorphum in trairas of the species Hoplias malabaricus in the central region of the Rio Grande do Sul State, Brazil. Were necropsied two parasites coming from a lake in the municipality of Cachoeira do Sul (Zoo), in which were found inside black muscle cysts. Within each cyst, there was a metacercariae, which was collected, fixed in AFA and stained for subsequent mounting permanent slides with canada balsam. The metacercariae were identified as belonging to the species I. dimorphum. The adult parasite is found in birds of the Southern and Center-West Brazil. Probably these fish were infected due to the presence of migratory birds in the zoo lake during certain periods of the year. Due to the circumstances, it is concluded that I. dimorphum is a parasite of trairas.

Keywords: Hoplias malabaricus. Ithyoclinostomum dimorphum. Parasitism.

O gênero Hoplias possui grande capacidade de adaptação a diferentes condições hidrológicas, sendo um peixe de ampla distribuição geográfica nas regiões tropicais e subtropicais do Brasil. É popularmente conhecido como traíra, trairão, taraíra, tararira, sendo a espécie Hoplias malabaricus a mais estudada nas populações das diversas bacias hidrográficas (1). Pesquisa relataram nessa espécie de peixe o parasitismo por Diplostomum (Austrodiplostomum) compactum e acantocéfalos do gênero Quadrigyrus nas planícies do Rio Paraná (2).

No Brasil, foi registrada a ocorrência da forma adulta de Ithyoclinostomum dimorphum no esôfago de aves da espécie Ardea cocoi, Nyctiocorax sp. e Tigrisoma brasiliense (3), os peixes atuam no ciclo deste trematoda como hospedeiros intermediários contendo a metacercária encistada na musculatura, brânquias, pericárdio, parede externa e esôfago, que apresentam corpo bastante alongado, estreito, achatado com comprimento e largura variado (2). O objetivo deste trabalho foi relatar o parasitismo por Ithyoclinostomum dimorphum em traíras da espécie Hoplias malabaricus na região central do Rio Grande do Sul.

Foram necropsiados dois exemplares de H. malabaricus oriundos de um lago no município de Cachoeira do Sul, RS (Zoológico), nos quais foram encontrados no interior da musculatura cistos pretos. Com a ruptura dos cistos observouse a presença de metacercárias, que foram coletadas, fixadas em AFA e coradas (4), para posterior montagem em lâminas permanentes com bálsamo do Canadá. Outra técnica utilizada foi a de hematoxilina de delafield, para facilitar a visualização das estruturas internas do trematoda (5). A identificação do parasita foi baseada em chave de classificação (2).

As metacercárias foram identificadas como pertencentes à espécie Itbyoclinostomum dimorphum. Metacercárias deste parasita já foram descritas por outros autores em peixes da espécie Schizodon borelli, oriundos do Rio Paraná (6). Semelhante a este estudo, a literatura cita a ocorrência de I. dimorphum em traíras coletadas no Rio Paraná (2). Com base nos trabalhos citados anteriormente, observou-se que a distribuição deste parasita no Brasil ocorre preferencialmente na região Sul.

O I. dimorphum adulto é encontrado em aves, sendo relatado por pesquisadores em aves da espécie Ardea cocoi na Região do Alto Paraná e em pássaros da família Ciconiiformes na região Centro-Oeste do Brasil $(7,8)$. Sabendo que as aves migratórias (hospedeiros definitivos) são as responsáveis pela disseminação deste digenético, sendo que neste estudo suspeita-se que estas foram as responsáveis pela ocorrência deste parasita na região central do Rio Grande do Sul. Pelas circunstâncias, conclui-se que o Ithyoclinostomum dimorphum é um parasita de traíras, assim como de aves silvestres na região Sul. 


\section{REFERÊNCIAS}

1. Gonçalves PC, Morelli S. Estudo cariotípico da população de Hoplias malabaricus do Rio das Pedras [online] 2000 [citado 12 jun. 2007]. Disponível em: http://www.propp.ufu.br/pub/B

2. Pavanelli GC, Schaeffer GV, Santos MS. Ocorrência e histopatologia de Ithyoclinostomum dimorphum (Diesing, 1850) (Trematoda: Clinostomatidae) em traíras coletadas no rio Paraná. Revista Unimar. 1990;12(1):69-75.

3. Travassos L, Freitas JFT, Kohn A. Trematódeos do Brasil. Memórias do Instituto Oswaldo Cruz. 1969;67(4):881-6.

4. Pinto C. Zoo-Parasitas de interesse médico e veterinário. Rio de Janeiro: Scientífica; 1945.

5. Eiras JC, Takemoto RM, Pavanelli GC. Métodos de estudo e técnicas laboratoriais em parasitologia de peixes. Maringá: EDUEM; 2000.

6. Machado MH, Pavanelli GC, Takemoto RM. Structure and diversity of endoparasitic infracommunities and the trophic level of Pseudoplatystoma corruscans and Schizodon Borelli (Osteichthyes) of the high Paraná river. Memórias do Instituto Oswaldo Cruz. 1996;91(4):441-8.

7. Dias MLGG, Santos MJ, Souza GTR, Machado MH, Pavanelli GC. Scaning electron microscopy of Ithyoclinostomum dimorphum (Trematoda: Clinostomidae), a parasite of Ardea cocoi (Aves: Ardeidea). Parasitology Research. 2003;90(3):355-8.

8. Pinto RM, Barros LA, Tortelly L, Teixeira RF, Gomes DC. Prevalence and pathology of helments of ciconiiforms birds from the brazilian swamplands. Journal Helmintology. 2004;78(3):259-64.

Recebido: 30/06/2007

Received: 06/30/2007

Aprovado: 10/11/2007

Approved: 11/10/2007 